\title{
Construction of Expression Shuttle Vectors for the Haloarchaeon Natrinema sp. J7 Based on Its Chromosomal Origins of Replication
}

\author{
Yuchen Wang, ${ }^{1}$ Beibei Chen, ${ }^{1}$ Linshan Sima, ${ }^{1}$ Mengzhuo Cao, ${ }^{1}$ and Xiangdong Chen ${ }^{1,2}$ \\ ${ }^{1}$ State Key Laboratory of Virology, College of Life Sciences, Wuhan University, Wuhan 430072, China \\ ${ }^{2}$ Hubei Provincial Cooperative Innovation Center of Industrial Fermentation, Wuhan 430072, China \\ Correspondence should be addressed to Xiangdong Chen; xdchen@whu.edu.cn
}

Received 20 August 2016; Revised 28 November 2016; Accepted 26 December 2016; Published 1 March 2017

Academic Editor: Toshiaki Fukui

Copyright (C) 2017 Yuchen Wang et al. This is an open access article distributed under the Creative Commons Attribution License, which permits unrestricted use, distribution, and reproduction in any medium, provided the original work is properly cited.

Haloarchaeon Natrinema sp. J7, the first reported archaeon harboring both plasmid and chromosome-based temperate viruses, is a useful model for investigating archaeal virus-host and virus-virus interactions. However, the lack of genetic tools has limited such studies. On the basis of the automatically replicating sequences of the $\mathrm{J} 7$ chromosome and the pyrF marker, we constructed seven vectors, six of which were confirmed to possess replication ability in a pyrF-deletion derivative of J7 (J7-F). Among these vectors, $\mathrm{pFJ1}, \mathrm{pFJ} 4$, and $\mathrm{pFJ} 6$ could be transformed into the host strain with relatively high efficiency (approximately $10^{3}$ colonyforming units $/ \mu \mathrm{g}$ DNA) and were present at about one copy per chromosome. These three vectors could be stably maintained in J7-F without selection and were used for heterologous protein expression. Only pFJ6 was found to be present in the transformed cells in an exclusively episomal, nonintegrated state (one copy per chromosome). In contrast, some pFJ1 and pFJ4 DNA was probably integrated into the J7-F chromosome. In addition, pFJ6 was found to be compatible with pYCJ in J7 cells, suggesting that these two vectors could be used for further studies of virus-virus and virus-host interactions.

\section{Introduction}

Extremely halophilic archaea constitute a group of microorganisms that thrive in hypersaline environments, including solar salterns and natural salt lakes. This group consists of approximately 48 genera and 177 species [1], forming an important part of archaea [2]. Compared to other groups of archaea, these haloarchaea are particularly suitable for research purposes, as they are easily cultured and manipulated in the laboratory. They also serve as useful models for studying archaeal viruses, which exhibit striking morphological diversity and unique gene contents. However, the study of these haloarchaea and their viruses is limited by the availability of appropriate genetic tools. Among the 48 genera of haloarchaea, genetic tools have been developed for only three genera, namely, Haloferax [3, 4], Halobacterium [5], and Haloarcula [6]. Considering that no single archaeal species is representative of the domain as a whole, or even its own specific group, it will be important to develop genetic tools for the many haloarchaea that still lack such resources.

Most haloarchaeal shuttle vectors are derived from endogenous plasmids, such as pGRB1 [7], pHH1 [8], and pNRC100 [9] from Halobacterium salinarum or pHK2 [4, 10], pHV2 [11, 12], and pHV1/4 [13] from Haloferax volcanii. The replicative function of some shuttle vectors originates from phage elements or chromosomal replication origins (oriCs), such as pUBP1 and pUBP2. These are constructed based on the $\Phi H$ replicon [5] and pBBori7, which was created using oriC/orc7 from Halobacterium sp. NRC-1 [14]. Most haloarchaea with established genetic tools can be transformed using polyethylene glycol (PEG). However, only a few selectable markers are available for haloarchaea. Novobiocin, which inhibits DNA gyrase $($ gyrB) [15], and mevinolin, which inhibits HMG-CoA reductase [16] are two widely used antibiotics in archaea. Auxotrophic selectable markers involved in amino acid or nucleotide biosynthesis 
have also been used. These include the ura3 and pyrE2 genes involved in uracil biosynthesis $[17,18]$, the $\operatorname{trp} A$ gene involved in tryptophan biosynthesis, the leuB gene participating in leucine biosynthesis, and the $h d r B$ gene used in thymidine biosynthesis [12]. Among these markers, ura3 and pyrE2 are particularly useful for gene knockout or replacement studies because they can be counterselected using 5-fluoroorotic acid [18].

The halobacterial genus Natrinema gen. nov., was proposed by McGenity et al. based on the phylogenetic analysis of the 16S rRNA gene sequences and taxonomic properties of its member species. Strains in this genus require that the salt concentration be at least $10 \%$ to allow for basic cell growth and $19.8 \%$ to $25.1 \%$ for optimal growth [19]. Seven species have been discovered in this genus so far. Among them is Natrinema sp. J7, which was isolated in a Yingcheng salt mine in Hubei province, China. It is the only strain for which genetic manipulation tools had been established $[20,21]$ and whose genome had been sequenced [22]. Studies of J7 derivatives have focused on the proteases SptA $[23,24]$ and SptC [25], heat shock protein $70[26,27]$, and DNA fragments conferring promoter activity in the three domains of life. Notably, one of its derivatives, J7-1, was the first archaeon reported to harbor two kinds of temperate haloarchaeal viruses: sphaerolipovirus SNJ1 [28, 29] and pleolipovirus SNJ2 [30]. Accordingly, J7-1 could be used as an excellent model for studying virus-host and virus-virus interactions in archaea. However, the lack of genetic tools hampered these studies.

Previously, integrative plasmids based on the isolated auxotrophic mutants of $\mathrm{J7}$ derivatives were constructed and used to express exogenous proteins in J7 [20]. Recently, a shuttle vector ( $\mathrm{pYCJ}$ ) based on the SNJ1 replicon (nucleotides 1-4481) was also constructed and validated for the expression and purification of heterologous proteins [21]. A number of ORFs and genetic elements controlling virus genome replication, maintenance, and copy number were identified in the SNJ1 replicon. Moreover, the key elements responsible for superinfection exclusion and lytic/lysogenic conversion of the SNJ1 virus are also located in this region (unpublished data). Because any mutation to the SNJ1 replicon would impair the self-replicating ability of the plasmid, the pYCJ vector is not suitable for investigating these regulators and elements. Thus, additional genetic tools must be developed to enable research on these viruses and their host. In this study, seven shuttle vectors derived from the predicted oriCs in the $\mathrm{J} 7$ chromosome were constructed. Each vector contained the $p y r F$ gene and the autonomously replicating sequence (ARS, one or two predicted oriCs). Six of these vectors (pFJ1, pFJ3, pFJ4, pFJ5, pFJ6, and pFJ7) were successfully transformed into J7-F, but only pFJ1, pFJ4, and pFJ6 could be transformed with relatively high efficiency $\left(10^{3}\right.$ colonyforming units $[\mathrm{cfu}] / \mu \mathrm{g}$ DNA). These three shuttle vectors were studied further and found to be stably maintained at approximately one copy per chromosome in J7-F without selection. pFJ1 and pFJ4 may exist either in the plasmid form or integrated into the host chromosome, whereas pFJ6 replicated only in a nonintegrated state and was compatible with pYCJ in J7 cells. All three vectors were validated for stable expression of the amylase protein in J7-F cells, making them instrumental for further studies on the SNJ1 virus, as well as J7 derivatives.

\section{Materials and Methods}

2.1. Strains, Media, and Growth Conditions. Strains used in this study are listed in Table 1. Different derivatives of Natrinema sp. J7 were grown in Halo-2 medium at $45^{\circ} \mathrm{C}$, as previously described [28, 30]. A J7-F strain lacking the chromosomal pyrF gene was a gift from Professor Yuping Huang (College of Life Science, Wuhan University). Minimal medium (MM; 18\%) and modified growth medium (MGM; $18 \%)$ were prepared as described in the Halohandbook [31]. Escherichia coli strains $\mathrm{DH} 5 \alpha$ and JM110 were used for plasmid DNA construction and were routinely cultured in Luria-Bertani medium at $37^{\circ} \mathrm{C}$. Agar (1.5\%) was added when solid analogs of the above-mentioned media were needed.

2.2. Predicting oriCs in the J7 Chromosome. The web-based tool Ori-Finder 2 was employed to predict oriCs in archaeal genomes [32]. OriCs were predicted based on an integrated analysis of base-composition asymmetry using the Z-curve method, the distribution of origin recognition boxes (ORBs) identified using Find Individual Motif Occurrence [33], and the occurrence of genes that are frequently close to oriCs.

2.3. Plasmid Construction. Plasmids and primers used in this study are listed in Tables 1 and 2, respectively. A $0.95 \mathrm{~kb}$ BamHI-SnaBI fragment consisting of the pyrF gene and the 200 bp promoter region of the $f d x$ gene $\left(\mathrm{P}_{f d x}\right)$ from Haloferax volcanii DS2 was cloned from the $\mathrm{pNBK}-\mathrm{F}$ plasmid into the BamHI and SnaBI sites of the pUC-M vector to generate pUC-M-pyrF.

Seven SnaBI-MfeI-digested ARSs containing one or two predicted oriCs amplified from the J7 genome were ligated into the digested pUC-M-pyrF plasmid to create pFJ1, pFJ2, pFJ3, pFJ4, pFJ5, pFJ6, and pFJ7. The specific locations of the 12 predicted oriCs and seven ARSs are shown in Table 3. A $1.7 \mathrm{~kb}$ fragment encompassing $a m y H$ and its 200 bp promoter (Apro-amyH) was amplified from Haloarcula hispanica DSM4426. Restriction enzyme sequences for MfeI, AflII, SpeI, and NotI were added to the $5^{\prime}$ end of the fragment and NotI, NheI, NsiI, and SphI sites were added to the $3^{\prime}$ end. The MfeI-Apro-amyH-SphI fragment was ligated into pFJ1, pFJ4, and pFJ6 to generate pFJ1$\mathrm{A}, \mathrm{pFJ} 4-\mathrm{A}$, and pFJ6-A, respectively. After digestion with NotI followed by self-ligation, the Apro-amyH fragment was

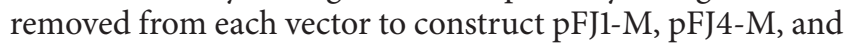
pFJ6-M. The physical maps of pFJ1-M, pFJ4-M, and pFJ6-M are shown in Figure 1. A $200 \mathrm{bp}$ fragment encompassing the upstream region of the 3916 gene (3916 pro) located in the J7 chromosome was amplified and ligated into pFJ1-M, pFJ4$\mathrm{M}$, and pFJ6-M to generate the plasmids pFJ1-M-3916, pFJ4M-3916, and pFJ6-M-3916, respectively, which were used for plasmid copy number determination. 
TABLE 1: Strains and plasmids used in this study.

\begin{tabular}{|c|c|c|}
\hline Strain & Description & Source \\
\hline Natrinema sp. J7-1 & With SNJ1 proviral genome $\mathrm{pHH} 205$, cannot be infected by SNJ1 & {$[28,34]$} \\
\hline Natrinema sp. J7-F & $\Delta p y r F$, can be infected by SNJ1 & $\begin{array}{l}\text { Yuping Huang, Wuhan } \\
\text { University, Wuhan, China }\end{array}$ \\
\hline Escherichia coli DH5 $\alpha$ & $\begin{array}{c}\text { SupE44 } \Delta \text { lacU169( } \varphi 80 \text { lacZ } \Delta \mathrm{M} 15) \text { hsdR17 recA1 endA1 gyrA96 thi-1 } \\
\text { relA1 }\end{array}$ & CCTCC \\
\hline Escherichia coli JM110 & $\begin{array}{c}\text { dam dcm supE44 hsdR17 thi leu rpsL lacY galK galT ara tonA thr tsx } \\
\left.\Delta(\text { lac-pro } \mathrm{AB}) \mathrm{F}^{\prime} \text { (traD36 pro } \mathrm{AB}^{+} \text {lacI }^{\mathrm{q}} \text { lacZ } \Delta \mathrm{M} 15\right)\end{array}$ & ССТCC \\
\hline \multicolumn{3}{|c|}{${ }^{2}$} \\
\hline pNBK-F & AmpR, MevR, used to clone $\mathrm{P}_{\mathrm{fdx}}-p y r F$ resistance fragment & $\begin{array}{l}\text { Yuping Huang, Wuhan } \\
\text { University, Wuhan, China }\end{array}$ \\
\hline pUC-M & pUC19 with SnaBI, MfeI, AgeI inserted between BamHI and Xbal & Our lab \\
\hline pUC-M-pyrF & $\begin{array}{l}\text { pUC-M with the insertion of } 0.95-\mathrm{kb} \text { BamHI-SnaBI } \mathrm{P}_{\mathrm{fdx}}-\text { pyrF } \\
\text { resistance fragment }\end{array}$ & This study \\
\hline pFJ1 & $\begin{array}{c}\text { pUC-M-pyrF with the insertion of the } 2.8-\mathrm{kb} \text { SnaBI-MfeI predicted } \\
\text { chromosomal ARS-1 }\end{array}$ & This study \\
\hline $\mathrm{pFJ} 2$ & $\begin{array}{c}\text { pUC-M-pyrF with the insertion of the } 3.3-\mathrm{kb} \text { SnaBI-MfeI predicted } \\
\text { chromosomal ARS-2 }\end{array}$ & This study \\
\hline $\mathrm{pFJ} 3$ & $\begin{array}{l}\text { pUC-M-pyrF with the insertion of the } 3.2-\mathrm{kb} \text { SnaBI-MfeI predicted } \\
\text { chromosomal } \mathrm{r} \text { ARS-3 }\end{array}$ & This study \\
\hline pFJ4 & $\begin{array}{c}\text { pUC-M-pyrF with the insertion of the } 2.2-\mathrm{kb} \text { SnaBI-MfeI predicted } \\
\text { chromosomal ARS-4 }\end{array}$ & This study \\
\hline pFJ5 & $\begin{array}{l}\text { pUC-M-pyrF with the insertion of the } 2.3-\mathrm{kb} \text { SnaBI-MfeI predicted } \\
\text { chromosomal ARS-5 }\end{array}$ & This study \\
\hline pFJ6 & $\begin{array}{c}\text { pUC-M-pyrF with the insertion of the } 2.0-\mathrm{kb} \text { SnaBI-MfeI predicted } \\
\text { chromosomal ARS-6 }\end{array}$ & This study \\
\hline pFJ7 & $\begin{array}{c}\text { pUC-M-pyrF with the insertion of the 1.1-kb SnaBI-MfeI predicted } \\
\text { chromosomal ARS-7 }\end{array}$ & This study \\
\hline pFJ1-Apro-amyH & $\begin{array}{c}\text { pFJ1 with the insertion of 1.5-kb MfeI-SphI Apro-amyH fragment } \\
\text { containing AflII, SpeI, NotI, NheI, and NsiI }\end{array}$ & This study \\
\hline pFJ1-M & pFJ1-Apro-amyH digested with NotI and then ligated by itself & This study \\
\hline pFJ4-Apro-amyH & $\begin{array}{l}\text { pFJ4 with the insertion of 1.5-kb MfeI-SphI Apro-amyH fragment } \\
\text { containing AflII, SpeI, NotI, NheI, and NsiI }\end{array}$ & This study \\
\hline pFJ4-M & pFJ4-Apro-amyH digested with NotI and then ligated by itself & This study \\
\hline pFJ6-Apro-amyH & $\begin{array}{l}\text { pFJ6 with the insertion of 1.5-kb MfeI-SphI Apro-amyH fragment } \\
\text { containing AflII, SpeI, NotI, NheI, and NsiI }\end{array}$ & This study \\
\hline pFJ6-M & pFJ6-Apro-amyH digested with NotI and then ligated by itself & This study \\
\hline pFJ1-M-pro3916 & pFJ1-M with the insertion of 200-bp NsiI-SphI pro3916 & This study \\
\hline pFJ4-M-pro3916 & pFJ4-M with the insertion of 200-bp NsiI-SphI pro3916 & This study \\
\hline pFJ6-M-pro3916 & pFJ6-M with the insertion of 200-bp NsiI-SphI pro3916 & This study \\
\hline
\end{tabular}

2.4. Transformation Method. Genetic transformation of J7F cells was performed at room temperature using PEG, as previously described [21]. MM (18\%) was prepared and used to select and propagate the Natrinema sp. J7-F transformants.

2.5. Stability and Maintenance of the Shuttle Vectors. To assess the structural stability of the vectors, we isolated pFJ1, pFJ4, and pFJ6 from J7-F transformants, back-transformed them into E. coli for amplification, extracted the plasmids, and subjected them to restriction enzyme digestion. The plasmid DNA was extracted from E. coli (without being transformed into J7-F cells), digested, and used as a positive control.

Maintenance of the pFJ1, pFJ4, and pFJ6 shuttle vectors was evaluated by calculating the rate at which each plasmid was lost per generation during nonselective growth, as previously described [21]. Briefly, selected clones were cultured to exponential phase for approximately five days at $45^{\circ} \mathrm{C}$ in $5 \mathrm{~mL} \mathrm{18 \%} \mathrm{MM.} \mathrm{The} \mathrm{cultures} \mathrm{were} \mathrm{then} \mathrm{diluted} \mathrm{1:100} \mathrm{in} 5 \mathrm{~mL}$ of fresh Halo- 2 medium and incubated for $24 \mathrm{~h}$. This dilution process was repeated 12 times (the doubling time of $\mathrm{J} 7$ cells was $\sim 2.9 \mathrm{~h}$ ). Every two dilutions, the cultures were spread onto $18 \%$ MGM plates (nonselective condition for J7-F cells). 
TABLE 2: Primers used in this study.

\begin{tabular}{|c|c|c|}
\hline Primers & $5^{\prime}-3^{\prime}$ sequence & Restriction sites \\
\hline $\mathrm{P}_{\mathrm{fdx}}-\mathrm{pyrF}-\mathrm{F}$ & AATGGATCCATCTCGGCTTATTCTTTTGATT & BamHI \\
\hline$P_{f d x}-p y r F-R$ & TAATACGTATTATTCTCGATACTGATTGAGTCGCTTC & SnaBI \\
\hline PARS-1-fwd & AATTACGTACGCCCCCGGTGCCTCCTCTCGGA & SnaBI \\
\hline PARS-1-rev & ATTCAATTGACTCGCCGCCGACTACCTCCCCGTCG & $M f e \mathrm{I}$ \\
\hline PARS-2-fwd & AATTACGTATAGCCCGGGAAATACTATCTTTGAGTTCT & SnaBI \\
\hline PARS-2-rev & ATTCAATTGGATCGACGCTGGGATATGAAAAGC & $M f e \mathrm{I}$ \\
\hline PARS-3-fwd & AATTACGTAAACGGCTTTCGGATCGAAAGCAGC & SnaBI \\
\hline PARS-3-rwd & ATTCAATTGTTCGGTCTGCGGTCCCCATTTCC & $M f e \mathrm{I}$ \\
\hline PARS-4-fwd & AATTACGTAGACACACACCACTGTTGCAAGTGAAG & SnaBI \\
\hline PARS-4-rev & ATTCAATTGGTGGCCGCACAAGATCGA & $M f e \mathrm{I}$ \\
\hline PARS-5-fwd & AATTACGTACGATCGTGCCGACGTTACCCGGT & SnaBI \\
\hline PARS-5-rev & ATTCAATTGCGATCCCGAAGACGACCGCGT & $M f e \mathrm{I}$ \\
\hline PARS-6-fwd & AATTACGTAGGAGACGGTCAGAGTTACTGGTCAGT & SnaBI \\
\hline PARS-6-rev & TAACAATTGCAAGGGTTCGTCTGAAACCGTGT & $M f e \mathrm{I}$ \\
\hline PARS-7-fwd & ATATACGTAAGGCTGACTGTATGCGAGT & SnaBI \\
\hline PARS-7-rev & ATACAATTGGCACGACAGTAACAGT & MfeI \\
\hline 3916pro(R)-F & AATATGCATGAGTAAAGTTCGTGTTTCCTTGATTA & NsiI \\
\hline 3916pro(R)-R & ATAGCATGCCGGACAAGACGCCCATTTG & SnaBI \\
\hline Apro-AmyH-M-F & AATCAATTGCTTAAGACTAGTGCGGCCGCGGGAGCCGGAAACGCGGTAGAGATA & MfeI, AgeI, SpeI, NotI \\
\hline Apro-AmyH-M-R & AAGCATGCTATGCATAGCTAGCGCGGCCGCAAGGTAGTGGAAAGCGAGCCAGCGC & NotI, NsiI, NdeI, SphI \\
\hline pyrF test-F & CGATCACCGTCAACCCCTACATGG & l \\
\hline pyrF test-R & TACTGATTGAGTCGCTTCTTCAGTCGTTT & l \\
\hline MevR test-F & TCGCCTCCCTCGAAGTCGGCACCGT & / \\
\hline MevR test-R & GAACAACGGCGAAGAAAAGGCAGTCCA & / \\
\hline
\end{tabular}

Fifty random clones were selected and reseeded onto $18 \%$ MGM or $18 \%$ MM plates. The numbers of clones that grew on both kinds of plates were counted. Survival on $18 \% \mathrm{MM}$ plates indicated maintenance of the vector.

2.6. Southern Blot Analysis. Southern blot analysis was performed to determine whether the shuttle vectors ( $\mathrm{pFJ1}, \mathrm{pFJ} 4$, and $\mathrm{pFJ6}$ ) had integrated into the J7-F chromosome and to measure their copy numbers. J7-F cells transformed with pFJ1, pFJ4, or pFJ6 were cultivated in $200 \mathrm{~mL} 18 \% \mathrm{MM}$ and harvested in exponential phase. Their genomic DNA was isolated as previously described [34], digested at the only HindIII site in the shuttle vectors, and separated by $1 \%(\mathrm{w} / \mathrm{v})$ agarose gel electrophoresis. After denaturation in alkaline solution ( $1 \mathrm{M} \mathrm{NaCl}, 0.5 \mathrm{MNaOH})$, DNA samples were transferred to a positively charged nylon membrane. Subsequently, the DNA was fixed by incubation at $80^{\circ} \mathrm{C}$ for $2 \mathrm{~h}$ and probed using a $p y r F$ fragment labeled with a digoxigenin(DIG-) dUTP random primer, based on the instructions of the DIG-High Prime DNA Labeling and Detection Starter Kit I (Roche). To determine plasmid copy number, a 200 bp upstream fragment of the J7 3916 gene, present at one copy per chromosome, was amplified and ligated into pFJ1, pFJ4, and pFJ6. Then, total DNA isolated from J7-F/pFJ1-3916pro, J7-F/pFJ4-3916pro, and J7-F/pFJ6-3916pro was digested with Sau3AI and subjected to Southern blot analysis as described above, using the DIG-labeled 3916 pro sequence as a probe.
2.7. Amylase Activity Assay. Specific amylase activity of the supernatants from CJ7/pYCJ-Apro-amyH, CJ7-F/pFJ1-AproamyH, CJ7-F/pFJ4-Apro-amyH, and CJ7-F/pFJ6-AproamyH was measured as previously described [21]. One unit of amylase activity was defined as the quantity of amylase required to hydrolyze $1 \mathrm{mg}$ of starch in $1 \mathrm{~h}$.

\section{Results}

3.1. Analyzing and Cloning of Replication Regions of the J7 Chromosome. To construct shuttle vectors for J7 derivatives, oriCs in the J7 chromosome were predicted using the webbased Ori-Finder 2 tool. As shown in Table 3(a), 12 oriCs were predicted in the $\mathrm{J} 7$ chromosome; most of them had a relatively low GC content compared to that of the $\mathrm{J} 7$ chromosome (64\%) and all of them contained ORBs. Except for oriC3 and oriC10, the other oriCs were all adjacent to $c d c 6$ genes, encoding replication-initiating proteins. However, the locations of some oriC pairs, such as oriC1 and oriC2, oriC5 and oriC6, and oriC7 and oriC8, as well as oriC11 and oriC12, were very close and adjacent to the same $c d c 6$ gene (Table 3(a)). Five presumed ARSs containing one or two oriCs and their adjacent $c d c 6$ gene were amplified (Table 3(b)). These were then ligated into pUC-M-pyrF to construct the pFJ1, pFJ2, pFJ3, pFJ4, and pFJ5 shuttle vectors. In addition, oriC12 and oriC3, which were not adjacent to the $c d c 6$ gene, were also amplified and used to construct pFJ6 and pFJ7, respectively. As shown in Table 3(b), all 
TABLE 3: Characteristics of predicted oriCs and replication regions used for shuttle vector construction.

(a) Characteristic of predicted oriCs

\begin{tabular}{lcccc}
\hline OriCs & Locations & $\begin{array}{c}\text { Number of ORB } \\
\text { elements }\end{array}$ & GC content (\%) & \multicolumn{2}{c}{ Adjacent to $c d c 6$} \\
gene
\end{tabular}

(b) Characteristic and replication ability of the shuttle vectors

\begin{tabular}{lccccc}
\hline $\begin{array}{l}\text { Shuttle } \\
\text { vectors }\end{array}$ & ARSs & $\begin{array}{c}\text { OriCs } \\
\text { contained }\end{array}$ & $\begin{array}{c}\text { Chromosomal locations } \\
\text { of ARSs (positions) }\end{array}$ & $\begin{array}{c}\text { Containing } \\
\text { cdc6 gene }\end{array}$ & $\begin{array}{c}\text { Transformation } \\
\text { efficiency }\end{array}$ \\
\hline pFJ1 & 1 & 1,2 & $120,345-123,145$ & $\mathrm{Y}$ & $(1.7 \pm 0.6) \times 10^{3}$ \\
pFJ2 & 2 & 4 & $434,194-437,483$ & $\mathrm{Y}$ & 0 \\
pFJ3 & 3 & 5,6 & $1,277,883-1,281,049$ & $\mathrm{Y}$ & $(3.9 \pm 0.4) \times 10^{1}$ \\
pFJ4 & 4 & 7,8 & $2,211,560-2,213,764$ & $\mathrm{Y}$ & $(2.1 \pm 0.1) \times 10^{3}$ \\
pFJ5 & 5 & 9 & $2,572,200-2,574,543$ & $\mathrm{Y}$ & $<10$ \\
pFJ6 & 6 & 10 & $2,860,000-2,862,000$ & $\mathrm{~N}$ & $(2.2 \pm 0.5) \times 10^{3}$ \\
pFJ7 & 7 & 3 & $399,506-400,667$ & $\mathrm{~N}$ & $<10$ \\
\hline
\end{tabular}

${ }^{a}$ Colony-forming units/ $\mu \mathrm{g}$ DNA.

vectors except $\mathrm{pFJ} 2$ were successfully transformed into J7F cells. Transformation efficiency of pFJ1, pFJ4, and pFJ6 was approximately $10^{3} \mathrm{cfu} / \mu \mathrm{g}$ DNA, a little lower than that of the SNJ1 replicon-based vector, pYCJ [21]. Only a few transformants were obtained after transforming JF-7 cells with $\mathrm{pFJ} 3, \mathrm{pFJ} 5$, and $\mathrm{pFJ} 7$. These results indicate that at least six regions in the $\mathrm{J} 7$ chromosome possess replication ability, which is consistent with the presence of multiple replication origins in the chromosome of most haloarchaeal strains [35].

3.2. Maintenance and Structural Stability of the oriC-Based Vectors in J7-F Cells. To assess the structural stability of all shuttle vectors (pFJ1, pFJ3, pFJ4, pFJ5, pFJ6, and pFJ7) and verify their ability to replicate independently in J7-F cells, plasmid DNA was extracted from each vector transformant and back-transformed into E. coli (posttransformed plasmids), reisolated, and subjected to enzymatic digestion. Shuttle vectors extracted from E. coli (pretransformed into J7F cells) were used as positive controls. As shown in Figure 2, pretransformed (lanes 2) and posttransformed (lanes 4) plasmids showed similar digestion profiles, indicating that structural stability of all constructs was maintained in J7-F cells and that these six vectors replicated independently of the chromosome. As mentioned above, $\mathrm{pFJ} 3$, pFJ5, and $\mathrm{pFJ} 7$ could be successfully transformed into J7-F cells, but their transformation efficiencies were too low to be suitable for genetic manipulation of $\mathrm{J7}$ cells. Hence, these three vectors were excluded from further studies.

Maintenance of pFJ1, pFJ4, and pFJ6 was determined by calculating the survival frequencies on $18 \% \mathrm{MM}$ during nonselective growth. As shown in Figure 3, all vectors could be stably maintained in J7-F cells after culturing for 12 days $(\sim 100$ generations), indicating that all of them could be stably segregated into daughter cells during cell growth.

3.3. Presence of pFJ6 in Transformed Cells in an Episomal, Nonintegrated State. Southern blot analysis was performed to determine whether $\mathrm{pFJ} 1, \mathrm{pFJ} 4$, and $\mathrm{pFJ} 6$ integrated into the J7-F chromosome. Total DNA extracted from three independent J7-F transformants of each plasmid was digested with HindIII, separated by $1 \%(\mathrm{w} / \mathrm{v})$ agarose gel electrophoresis (Figure 4(a)), and then subjected to Southern blot analysis. Plasmids extracted from E. coli were used as controls and the DIG-labeled pyrF gene was used as probe. Only one HindIII restriction site was found in pFJ1, pFJ4, and pFJ6 plasmids. Thus, digested nonintegrated plasmids from total DNA samples were predicted to be linearized to $5.86 \mathrm{~kb}$, $5.66 \mathrm{~kb}$, and $5.35 \mathrm{~kb}$, respectively, distinguishing them from 


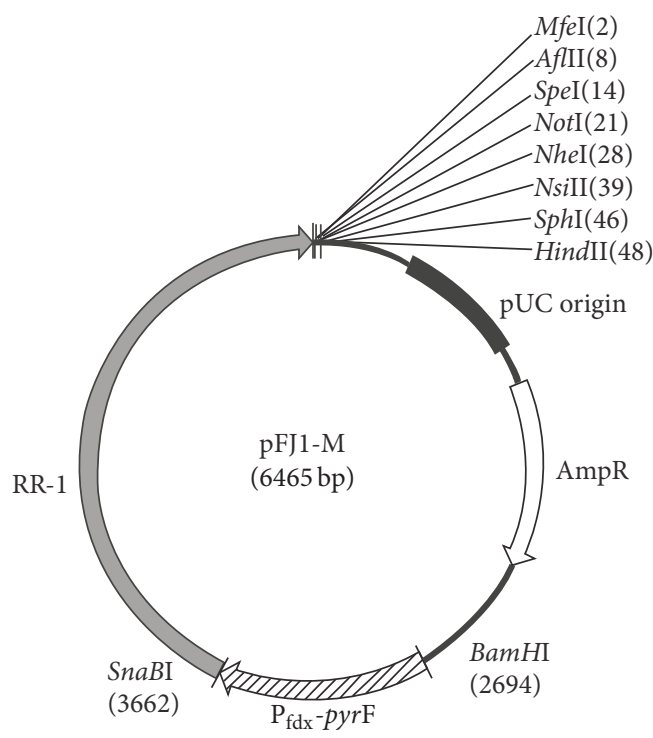

(a)

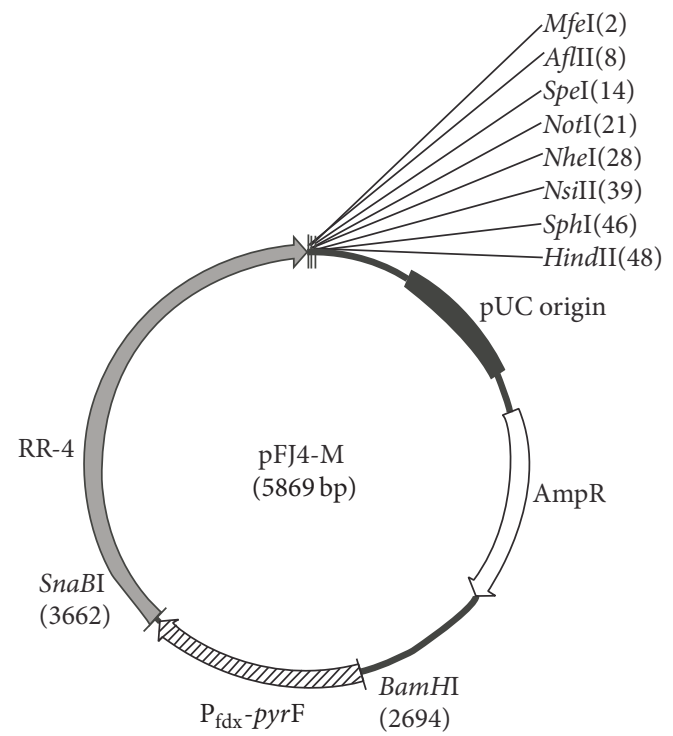

(b)

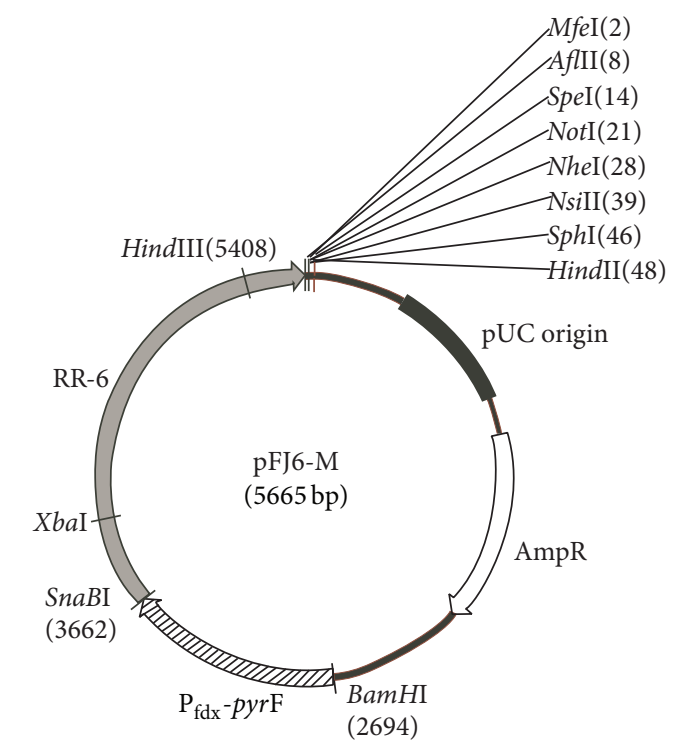

(c)

Figure 1: Physical maps of the pFJ1-M (a), pFJ4-M (b), and pFJ6-M (c) plasmids.

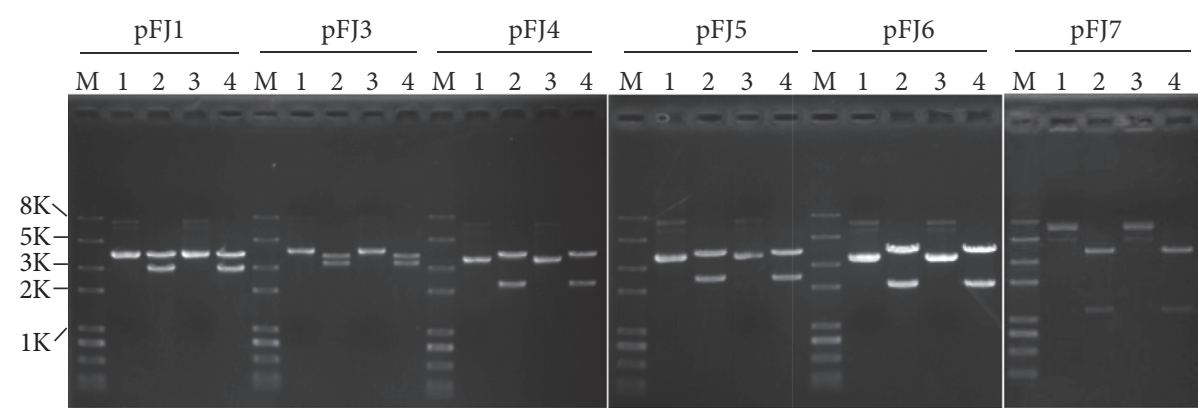

Figure 2: Structural stability of the shuttle vectors in J7-F cells. Pretransformed plasmids (extracted from E. coli before being transformed into J7-F, lanes 1 and 2) and back-transformed plasmid (extracted from E. coli following transformation into J7-F, lanes 3 and 4) were digested with restriction enzymes to compare their structural stabilities. M, 2 kb plus II marker; lanes 1 and 3, undigested plasmids; lanes 2 and 4 , plasmids digested with SnaBI and MfeI. 


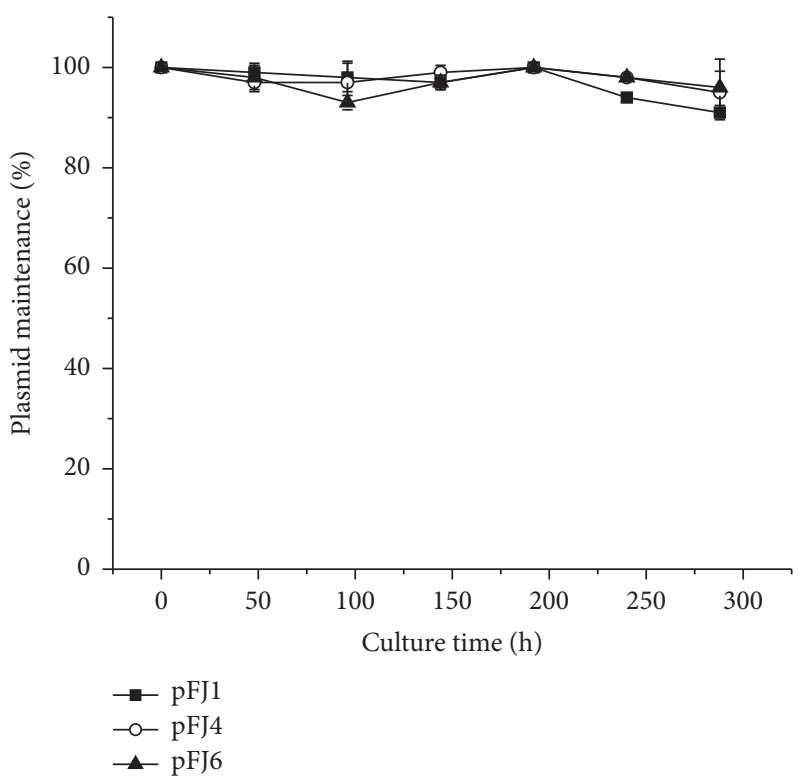

FIGURE 3: Maintenance of the pFJ1, pFJ4, and pFJ6 shuttle vectors in J7-F cells. J7-F cells containing different shuttle vectors $(50 \mu \mathrm{L})$ were diluted in $5 \mathrm{~mL}$ Halo-2 medium and grown for $24 \mathrm{~h}$. For every two dilutions (12 dilutions in total), aliquots were spread onto Halo-2 plates, and 50 random colonies were selected and spotted onto nonselective $18 \%$ MGM plates. Maintenance was measured by calculating the percentage of colonies growing on selective $18 \% \mathrm{MM}$ plates.

integrated plasmids. As shown in Figure 4(b), hybridization signals could be observed at every position corresponding to the linearized plasmid DNA, either in the HindIII-digested DNA samples extracted from E. coli (lanes 1, 5, and 9) or in the HindIII-digested total DNA preparations extracted from cultures of J7-F clones carrying pFJ1 (lanes 2-4), pFJ4 (lanes 6-8), and pFJ6 (lanes 10-12). However, compared with the single hybridization signal obtained using total DNA extracted from pFJ6-transformed J7-F cells (lanes 10-12), the probe also hybridized with a relatively large fragment in the total DNA samples extracted from pFJ1- and pFJ4transformed J7-F cells (lanes 2-4 and 6-8, resp.). These data indicate that only pFJ6 was present in an episomal, nonintegrated state in $\mathrm{J7}$ cells, whereas pFJ1 and pFJ4 may have integrated into the CJ7-F chromosome. Additional experiments are required to confirm these results.

3.4. Relative Copy Numbers of pFJ1, pFJ4, and pFJ6 in J7$F$ Cells. Shuttle vectors containing chromosome oriCs are usually present at one copy per chromosome [36]. We tested whether this was true for pFJ1, pFJ4, and pFJ6. The $200 \mathrm{bp}$ single-copy 3916 pro segment was amplified and ligated into the shuttle vectors used in this study. Total DNA from three randomly selected independent J7-F transformants containing pFJ1-M-3916, pFJ4-M-3916, and pFJ6-M-3916, respectively, was digested with Sau3AI, separated by $1 \%$ agarose gel electrophoresis, and subjected to Southern blot analysis using a DIG-labeled 3916 pro probe. A $686 \mathrm{bp}$ fragment containing 3916 pro was liberated from the J7 chromosome after digestion with Sau3AI, whereas $1119 \mathrm{bp}$,
$978 \mathrm{bp}$, and $1266 \mathrm{bp}$ fragments containing 3916 pro were generated from pFJ1-M-3916, pFJ4-M-3916, and pFJ6-M-3916 (Figure 5(a)). As shown in Figure 5(b), the DIG-labeled 3916 pro sequence hybridized to the $686 \mathrm{bp}$ fragment from the J7 chromosome and the corresponding fragment of each plasmid. The intensities of the hybridization signals generated from pFJ6-M-3916 and the chromosomal DNA fragment were almost identical, indicating that pFJ6 was present at approximately one copy per chromosome in J7-F cells. In contrast, the intensity of the hybridization signals generated by pFJ1-M-3916 and pFJ4-M-3916 was slightly stronger than that of the corresponding chromosomal DNA fragment, implying that pFJ1-M-3916 and pFJ4-M-3916 were present at more than one copy per chromosome. These results may be attributed to the fact that a portion of $\mathrm{pFJ} 1$ and $\mathrm{pFJ} 4$ could integrate into the J7 chromosome; however, additional experiments are required to confirm this thesis.

3.5. Utility of Shuttle Vectors pFJ1, pFJ4, and pFJ6. To determine the utility of these shuttle vectors, the Haloarcula hispanica DSM 4426 amy $\mathrm{H}$ gene and its promoter were inserted into pFJ1, pFJ4, and pFJ6. After transforming CJ7 cells with

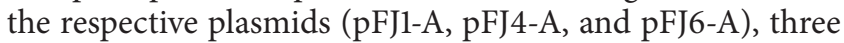
randomly selected colonies per plasmid were transferred onto $18 \%$ MGM plates supplemented with $2 \%$ (w/v) soluble starch. As shown in Figure 6(a), transparent halos were detected around the colonies after flooding the plates with iodine solution, suggesting that amylase was well expressed. In contrast, colonies of J7-F cells harboring only pFJ1, pFJ4, or pFJ6 showed no amylase activity, confirming the role of amylase in starch consumption. As shown in Figure 6(b), amylase activity in the supernatants of CJ7-F/pFJ1-Apro-amyH, CJ7F/pFJ4-Apro-amyH, and CJ7-F/pFJ6-Apro-amyH cultures at the same OD600 was generally lower than that of CJ7/pYCJApro-amyH. This result can be explained by the copy number of pYCJ (one to three copies per chromosome) [21] being higher than that of pFJ1, pFJ4, and pFJ6. The highest amylase activity occurred in CJ7-F/pFJ6-Apro-amyH, suggesting that $\mathrm{pFJ6}$ drove greater protein expression than $\mathrm{pFJ} 1$ and $\mathrm{pFJ} 4$.

3.6. Compatibility of pFJ6 and SNJ1 Replicon-Based pYCJ. Compatible shuttle vectors are excellent tools for investigating protein-protein and protein-DNA interactions in prokaryotes. Previously, we reported the first Natrinema sp. J7 E. coli shuttle vector, pYCJ, which was constructed based on the SNJ1 replicon and validated for stable expression of heterologous proteins. Because pFJ6 could also be maintained in $\mathrm{J} 7$ cells and did not integrate into the J7 chromosome, we tested whether pFJ6 and pYCJ were compatible with each other. pFJ6 and pYCJ were cotransformed into J7-F cells and transformants were selected on $18 \% \mathrm{MM}$ containing $5 \mu \mathrm{g} / \mathrm{mL}$ mevinolin. The presence of pFJ6 and pYCJ in the transformants was detected by PCR using primers targeting pyrF and mevR, respectively. The result showed that $p y r F$ and mevR were detected in all randomly selected transformants (data not shown), indicating that these two replicons were compatible with each other. 


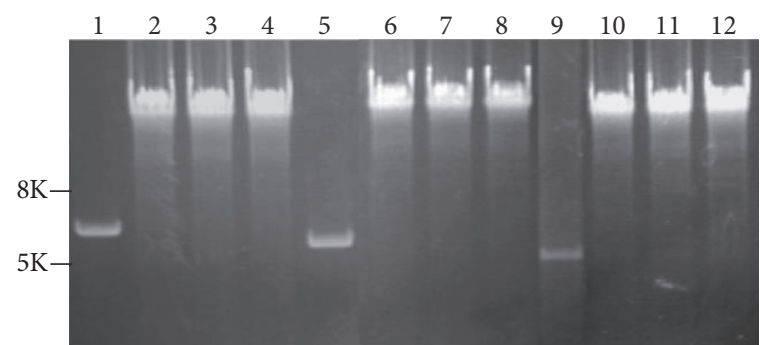

(a)

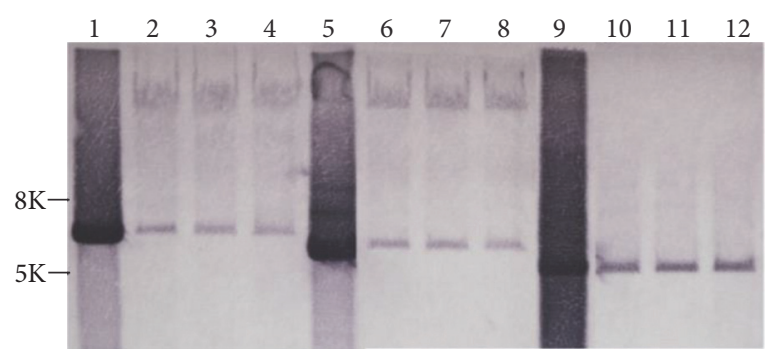

(b)

FIGURE 4: Determination of whether pFJ1, pFJ4, and pFJ6 could integrate into the J7 chromosome by Southern blot analysis. HindIII-digested total DNA extracted from cultures of J7-F clones carrying the pFJ1 (lanes 2-4), pFJ4 (lanes 6-8), or pFJ6 (lanes 10-12) plasmid were (a) separated on a $1 \%$ agarose gel at $20 \mathrm{~V}$ for $>12 \mathrm{~h}$ and then (b) subjected to Southern blot analysis. The pFJ1, pFJ4, and pFJ6 plasmids were propagated in E. coli, digested with HindIII, and loaded on lanes 1, 5, and 9 as controls. The DIG-labeled pyrF sequence was used as a hybridization probe.

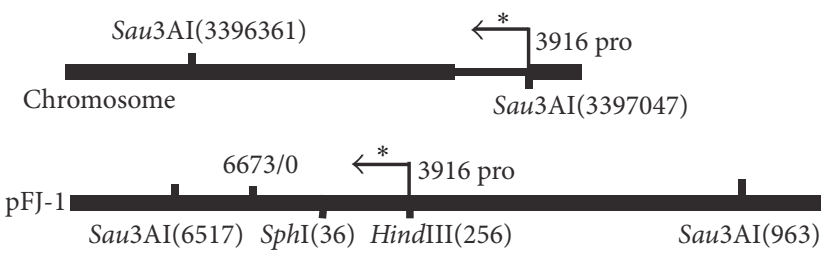

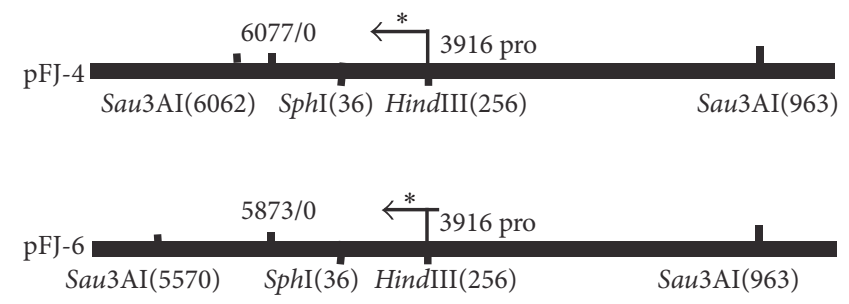

(a)

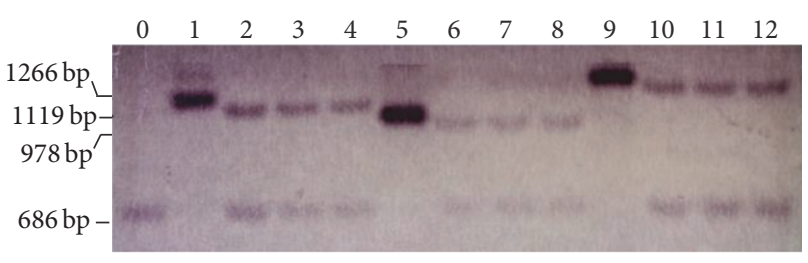

(b)

FIGURE 5: Relative copy numbers of the pFJ1, pFJ4, and pFJ6 plasmids in J7-F cells. (a) Schematic diagrams of the J7 chromosome and indicated vectors. The specific locations of Sau3AI sites in the J7 chromosome and plasmids are shown. The 200 bp 3916 pro segment, which was used as a DIG-labeled probe, is indicated with arrows and asterisks. (b) The copy numbers of pFJ1-pro3916, pFJ4-pro3916, and pFJ6-pro3916 were determined by Southern blot analysis. Sau3AI-digested total DNA from J7-F cells transformed with pFJ1 (lanes 2-4), pFJ4 (lanes 6-8), or pFJ6 (lanes 10-12) was hybridized with the DIG-labeled 3916 pro sequence. Total DNA samples from J7-F cells (lane 0), pFJ1-pro3916 (lane 1), pFJ4-pro3916 (lane 5), and pFJ6-pro3916 (lane 9) plasmids propagated in E. coli were used as controls.

\section{Discussion}

Haloarchaeon Natrinema sp. J7 is the first archaeon known to harbor both plasmid- and chromosome-based temperate viruses, SNJ1 and SNJ2. These two viruses display many interesting features. First, the efficient production of SNJ2 could only be achieved in J7 strains coinfected with SNJ1, indicating that SNJ1 promoted the replication of SNJ2 [30]. However, little is known about the mechanism behind this virus-virus interaction. Second, SNJ1 could infect CJ7, which does not harbor $\mathrm{pHH} 205$, but could not infect J7-1 (which does harbor pHH205), indicating that the lysogenic SNJ1 virus could establish superinfection exclusion or immunity, a phenomenon poorly understood in archaea. Third, several ORFs and genetic elements controlling virus genome replication, maintenance, and copy number were recently identified in the SNJ1 virus [21]. However, the mechanisms of these genetic elements and genes are completely unknown. All of these observations suggest that $\mathrm{J7}$ and its viruses are excellent models for studying virus-host and virus-virus interactions in archaea. To date, SNJ1 replicon-based pYCJ has been the only shuttle vector enabling genetic manipulation in J7 cells. Because the pYCJ shuttle vector contains the SNJ1 replicon [21], it could not be used for most studies on SNJ1, especially for functional studies on the regulatory proteins encoded by the SNJ1 replicon. In addition, given that only one shuttle vector was available for J7 cells, studies on protein-protein or protein-DNA interactions have also been limited. In this study, seven vectors based on the predicted oriCs in the J7 chromosome and the $p y r F$ marker were constructed; six of them could replicate in the uracil auxotrophic J7 strain (J7F). Three of these plasmids ( $\mathrm{pFJ1}, \mathrm{pFJ} 4$, and $\mathrm{pFJ} 6$ ) could be transformed into J7-F cells with high efficiency $\left(10^{3} \mathrm{cfu} / \mu \mathrm{g}\right.$ DNA). These three vectors were stably maintained in transformed J7-F cells without selection and could be used to express heterologous proteins. Notably, one of these vectors, 


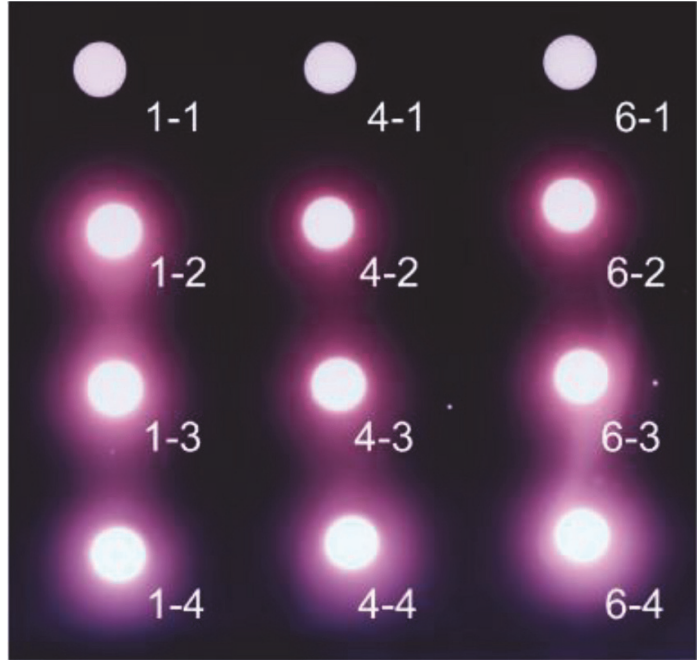

(a)

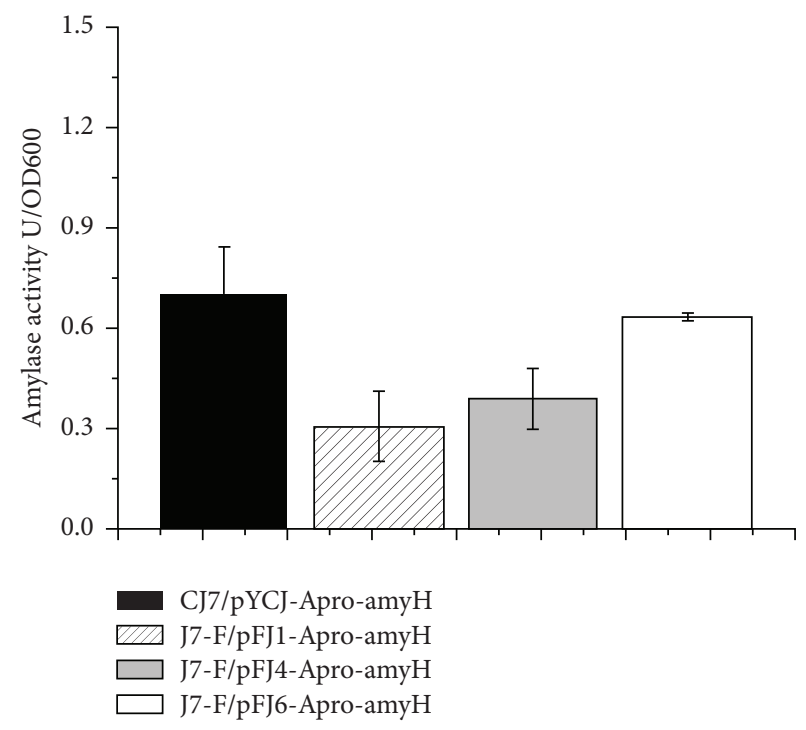

(b)

Figure 6: Amylase expression in J7-F cells using pFJ1, pFJ4, and pFJ6. (a) The pFJ1-A, pFJ4-A, and pFJ6-A plasmids were transformed into J7-F cells. Three random transformants were transferred to $5 \mathrm{~mL}$ of Halo-2 medium, grown to exponential phase, and $2 \mu \mathrm{L}$ of each culture was spotted onto $18 \%$ MGM plates supplemented with $2 \%(\mathrm{w} / \mathrm{v})$ soluble starch. After five days, iodine solution was added to the plates, and halos formed immediately around the selected transformants indicating that amylase was successfully expressed. 1-1: J7-F transformed with pFJ1 (negative control); 1-2, 1-3, and 1-4: J7-F transformants harboring pFJ1-A; 4-1: J7-F transformed with pFJ4 (negative control); 4-2, 4-3, and 4-4: J7-F transformants harboring pFJ4-A; 6-1: CJ7 cells transformed with pFJ6 (negative control); 6-2, 6-3, and 6-4: J7-F transformants harboring pFJ6-A (negative control). (b) Amylase-specific activities of supernatants collected from CJ7/pFJ1-Apro-amyH, CJ7/pFJ4-AproamyH, CJ7/pFJ6-Apro-amyH, and CJ7/pYCJ-Apro-amyH cultures. One unit of amylase activity was defined as the quantity of amylase required to hydrolyze $1 \mathrm{mg}$ of starch in $1 \mathrm{~h}$.

pFJ6, existed as a plasmid in $\mathrm{J7}$ cells and was compatible with pYCJ. These plasmids should serve as valuable tools for further studies on virus-virus and virus-host interactions in haloarchaea.

The vectors developed in this study displayed many advantages compared to pYCJ. First, they could stably replicate and segregate into daughter cells even under nonselective growth conditions. In contrast, pYCJ has been reported to disappear from CJ7 cells after three days without antibiotic selection [21]. This property is particularly important given that only a few markers are available for archaea. Second, the molecular weights of the vectors constructed here were all around $6 \mathrm{~kb}$, which is much smaller than that of most of the archaeal shuttle vectors previously reported [37], including pYCJ (about $9.9 \mathrm{~kb}$ ). Consequently, these shuttle vectors should be capable of accommodating larger exogenous fragments and will be much more convenient for genetic manipulation in general. Finally, given their compatibility with SNJ1 (pHH205) and pYCJ, the shuttle vectors constructed in this study and especially $\mathrm{pFJ} 6$ could be used for studying the regulators located in the SNJ1 replicon. This will be made possible by expressing only fragments or mutated regions of the SNJ1 replicon. Such kind of molecular manipulation could not be achieved using pYCJ, because any mutation in the 1-4481 region of SNJ1 would impair the stability of pYCJ [21]. In addition, the vectors constructed in this study will provide a useful tool for studying several biological processes in $\mathrm{J} 7$ cells. These include superinfection exclusion and lytic/lysogenic conversion of the SNJ1 virus, whose key determinants are located in the 1-4481 region of SNJ1 (unpublished data).

In addition to viral studies, the vectors developed here may be useful for investigating $\mathrm{J7}$ chromosome replication and segregation. Archaeal oriCs normally consist of a long intergenic sequence encompassing an A/T-rich, duplexunwinding element. They are typically located upstream of a $c d c 6 /$ orcl gene, which encodes a putative initiator protein that is homologous to Orcl of the eukaryotic ORC complex or the helicase loader Cdc6 $[38,39]$. Twelve oriCs were predicted in the $\mathrm{J} 7$ chromosome using Ori-Finder 2 software; most of them were adjacent to a putative $c d c 6$ gene. Six of the shuttle vectors containing one or two of the oriCs could replicate independently in J7-F cells, suggesting that at least six of the 12 predicted oriCs had the ability to initiate DNA replication. This is not surprising given that most archaea contain multiple replication origins [38]. However, it is remarkable that the homology between the nucleotide sequences in these six regions and the amino acid sequences of replication initiation proteins is low. It will be of interest to test which oriC in these shuttle vectors is required for replication and whether the adjacent $c d c 6$ gene is also essential. pFJ6 carrying the predicted oriC10 does not contain a putative $c d c 6$ gene, but it can replicate, suggesting that some of the $c d c$ genes may be responsible for replication initiation at multiple origins. Furthermore, three of the shuttle vectors are maintained at a single copy per chromosome without 
selection, indicating that their replication is coordinated with that of the chromosome and that they are faithfully segregated into daughter cells. Investigating the molecular mechanisms underlying such coordination will be particularly interesting because the control and coordination of replication initiation at multiple origins in archaea are poorly understood. These vectors are excellent tools for investigating this important question in archaea.

\section{Competing Interests}

The authors declare that there is no conflict of interests regarding the publication of this paper.

\section{Acknowledgments}

This work was supported by grants from the National Natural Science Foundation of China (no. 31570174), the National Fund for Fostering Talents of Basic Sciences (J1103513), and Research (Innovative) Fund of Laboratory of Wuhan University. The authors thank Dr. Shishen Du (University of Kansas Medical Center, USA) for many helpful discussions and for revising the manuscript.

\section{References}

[1] R. S. Gupta, S. Naushad, and S. Baker, "Phylogenomic analyses and molecular signatures for the class Halobacteria and its two major clades: a proposal for division of the class Halobacteria into an emended order Halobacteriales and two new orders, Haloferacales ord. nov. and Natrialbales ord. nov., containing the novel families Haloferacaceae fam. nov. and Natrialbaceae fam. nov," International Journal of Systematic and Evolutionary Microbiology, vol. 65, no. 3, pp. 1050-1069, 2015.

[2] C. R. Woese, O. Kandler, and M. L. Wheelis, "Towards a natural system of organisms: proposal for the domains archaea, bacteria, and eucarya," Proceedings of the National Academy of Sciences of the United States of America, vol. 87, no. 12, pp. 45764579, 1990.

[3] W. L. Lam and W. F. Doolittle, "Shuttle vectors for the archaebacterium Halobacterium volcanii," Proceedings of the National Academy of Sciences of the United States of America, vol. 86, no. 14, pp. 5478-5482, 1989.

[4] M. Holmes, F. Pfeifer, and M. Dyall-Smith, "Improved shuttle vectors for Haloferax volcanii including a dual-resistance plasmid," Gene, vol. 146, no. 1, pp. 117-121, 1994.

[5] U. Blaseio and F. Pfeifer, "Transformation of Halobacterium halobium: development of vectors and investigation of gas vesicle synthesis," Proceedings of the National Academy of Sciences of the United States of America, vol. 87, no. 17, pp. 6772-6776, 1990.

[6] S. W. Cline and W. F. Doolittle, "Transformation of members of the genus Haloarcula with shuttle vectors based on Halobacterium halobium and Haloferax volcanii plasmid replicons," Journal of Bacteriology, vol. 174, no. 3, pp. 1076-1080, 1992.

[7] M. P. Krebs, T. Hauss, M. P. Heyn, U. L. RajBhandary, and H. G. Khorana, "Expression of the bacterioopsin gene in Halobacterium halobium using a multicopy plasmid," Proceedings of the National Academy of Sciences of the United States of America, vol. 88, no. 3, pp. 859-863, 1991.
[8] F. Pfeifer and P. Ghahraman, "Plasmid pHH1 of Halobacterium salinarium: characterization of the replicon region, the gas vesicle gene cluster and insertion elements," MGG Molecular \& General Genetics, vol. 238, no. 1-2, pp. 193-200, 1993.

[9] W.-L. Ng and S. DasSarma, "Minimal replication origin of the 200-kilobase Halobacterium plasmid pNRC100," Journal of Bacteriology, vol. 175, no. 15, pp. 4584-4596, 1993.

[10] M. L. Holmes and M. L. Dyall-Smith, "A plasmid vector with a selectable marker for halophilic archaebacteria," Journal of Bacteriology, vol. 172, no. 2, pp. 756-761, 1990.

[11] R. L. Charlebois, W. L. Lam, S. W. Cline, and W. F. Doolittle, "Characterization of pHV2 from Halobacterium volcanii and its use in demonstrating transformation of an archaebacterium," Proceedings of the National Academy of Sciences of the United States of America, vol. 84, no. 23, pp. 8530-8534, 1987.

[12] T. Allers, H.-P. Ngo, M. Mevarech, and R. G. Lloyd, "Development of additional selectable markers for the halophilic archaeon Haloferax volcanii based on the leuB and trpA genes," Applied and Environmental Microbiology, vol. 70, no. 2, pp. 943953, 2004.

[13] C. Norais, M. Hawkins, A. L. Hartman, J. A. Eisen, H. Myllykallio, and T. Allers, "Genetic and physical mapping of DNA replication origins in Haloferax volcanii," PLOS genetics, vol. 3, no. $5,2007$.

[14] B. R. Berquis and S. DasSarma, "An archaeal chromosomal autonomously replicating sequence element from an extreme halophile, Halobacterium sp. strain NRC-1," Journal of Bacteriology, vol. 185, no. 20, pp. 5959-5966, 2003.

[15] M. L. Holmes and M. L. Dyall-Smith, "Mutations in DNA gyrase result in novobiocin resistance in halophilic archaebacteria," Journal of Bacteriology, vol. 173, no. 2, pp. 642-648, 1991.

[16] W. L. Lam and W. F. Doolittle, "Mevinolin-resistant mutations identify a promoter and the gene for a eukaryotelike 3-hydroxy-3-methylglutaryl-coenzyme A reductase in the archaebacterium Haloferax volcanii," Journal of Biological Chemistry, vol. 267, no. 9, pp. 5829-5834, 1992.

[17] R. F. Peck, S. DasSarma, and M. P. Krebs, "Homologous gene knockout in the archaeon Halobacterium salinarum with ura3 as a counterselectable marker," Molecular Microbiology, vol. 35, no. 3, pp. 667-676, 2000.

[18] G. Bitan-Banin, R. Ortenberg, and M. Mevarech, “Development of a gene knockout system for the halophilic archaeon Haloferax volcanii by use of the pyrE gene," Journal of Bacteriology, vol. 185, no. 3, pp. 772-778, 2003.

[19] T. J. McGenity, R. T. Gemmell, and W. D. Grant, "Proposal of a new halobacterial genus Natrinema gen. nov., with two species Natrinema pellirubrum nom. nov. and Natrinema pallidum nom. nov.," International Journal of Systematic Bacteriology, vol. 48, no. 4, pp. 1187-1196, 1998.

[20] J. Lv, S. Wang, Y. Wang, Y. Huang, and X. Chen, "Isolation and molecular identification of auxotrophic mutants to develop a genetic manipulation system for the haloarchaeon natrinema sp. J7-2," Archaea, vol. 2015, Article ID 483194, 16 pages, 2015.

[21] Y. Wang, L. Sima, J. Lv et al., "Identification, characterization, and application of the replicon region of the halophilic temperate sphaerolipovirus SNJ1, Journal of Bacteriology, vol. 198, no. 14, pp. 1952-1964, 2016.

[22] J. Feng, B. Liu, Z. Zhang et al., "The complete genome sequence of natrinema sp. J7-2, a haloarchaeon capable of growth on synthetic media without amino acid supplements," PLoS ONE, vol. 7, no. 7, Article ID e41621, 2012. 
[23] Z. Xu, X. Du, T. Li, F. Gan, B. Tang, and X.-F. Tang, "Functional insight into the C-terminal extension of halolysin SptA from haloarchaeon Natrinema sp. J7," PLoS ONE, vol. 6, no. 8, Article ID e23562, 2011.

[24] W. Shi, X.-F. Tang, Y. Huang, F. Gan, B. Tang, and P. Shen, "An extracellular halophilic protease SptA from a halophilic archaeon Natrinemasp. J7: gene cloning, expression and characterization," Extremophiles, vol. 10, no. 6, pp. 599-606, 2006.

[25] Y. Zhang, M. Wang, X. Du et al., "Chitin accelerates activation of a novel haloarchaeal serine protease that deproteinizes chitincontaining biomass," Applied and Environmental Microbiology, vol. 80, no. 18, pp. 5698-5708, 2014.

[26] H. Zhang, P. Cui, L. Lin, P. Shen, B. Tang, and Y.-P. Huang, "Transcriptional analysis of the hsp70 gene in a haloarchaeon Natrinema sp. J7 under heat and cold stress," Extremophiles, vol. 13, no. 4, pp. 669-678, 2009.

[27] W. Chen, G. Yang, Y. He et al., "Nucleotides flanking the start codon in $h s p 70$ mRNAs with very short 5'-UTRs greatly affect gene expression in haloarchaea," PLOS ONE, vol. 10, no. 9, Article ID e0138473, 2015.

[28] Z. Zhang, Y. Liu, S. Wang et al., "Temperate membranecontaining halophilic archaeal virus SNJ1 has a circular dsDNA genome identical to that of plasmid pHH205," Virology, vol. 434, no. 2, pp. 233-241, 2012.

[29] Y. Mei, C. He, Y. Huang et al., "Salinity regulation of the interaction of halovirus SNJ1 with its host and alteration of the halovirus replication strategy to adapt to the variable ecosystem," PLoS ONE, vol. 10, no. 4, Article ID e0123874, 2015.

[30] Y. Liu, J. Wang, Y. Liu et al., "Identification and characterization of SNJ2, the first temperate pleolipovirus integrating into the genome of the SNJ1-lysogenic archaeal strain," Molecular Microbiology, vol. 98, no. 6, pp. 1002-1020, 2015.

[31] M. Dyall-Smith, The Halohandbook: Protocols for Haloarchaeal Genetics, Haloarchaeal Genetics Laboratory, Melbourne, Austrulia, 2008.

[32] H. Luo, C.-T. Zhang, and F. Gao, "Ori-Finder 2, an integrated tool to predict replication origins in the archaeal genomes," Frontiers in Microbiology, vol. 5, 2014.

[33] C. E. Grant, T. L. Bailey, and W. S. Noble, "FIMO: scanning for occurrences of a given motif," Bioinformatics, vol. 27, no. 7, pp. 1017-1018, 2011.

[34] P. Shen and Y. Chen, "Plasmid from Halobacterium halobium and its restriction map," Yi Chuan Xue Bao, vol. 21, no. 5, pp. 409-416, 1993.

[35] Z. Wu, H. Liu, J. Liu, X. Liu, and H. Xiang, "Diversity and evolution of multiple orc/cdc6-adjacent replication origins in haloarchaea," BMC Genomics, vol. 13, no. 1, article no. 478, 2012.

[36] H. Atomi, T. Imanaka, and T. Fukui, "Overview of the genetic tools in the Archaea," Frontiers in Microbiology, vol. 3, 2012.

[37] J. Farkas, J. Picking, and T. Santangelo, "Genetic techniques for the archaea," Annual Review of Genetics, vol. 47, article 539, 2013.

[38] Z. Wu, J. Liu, H. Yang, and H. Xiang, "DNA replication origins in archaea," Frontiers in Microbiology, vol. 5, article no. 179, 2014.

[39] H. Wang, N. Peng, S. A. Shah, L. Huang, and Q. She, "Archaeal extrachromosomal genetic elements," Microbiology and Molecular Biology Reviews, vol. 79, no. 1, pp. 117-152, 2015. 

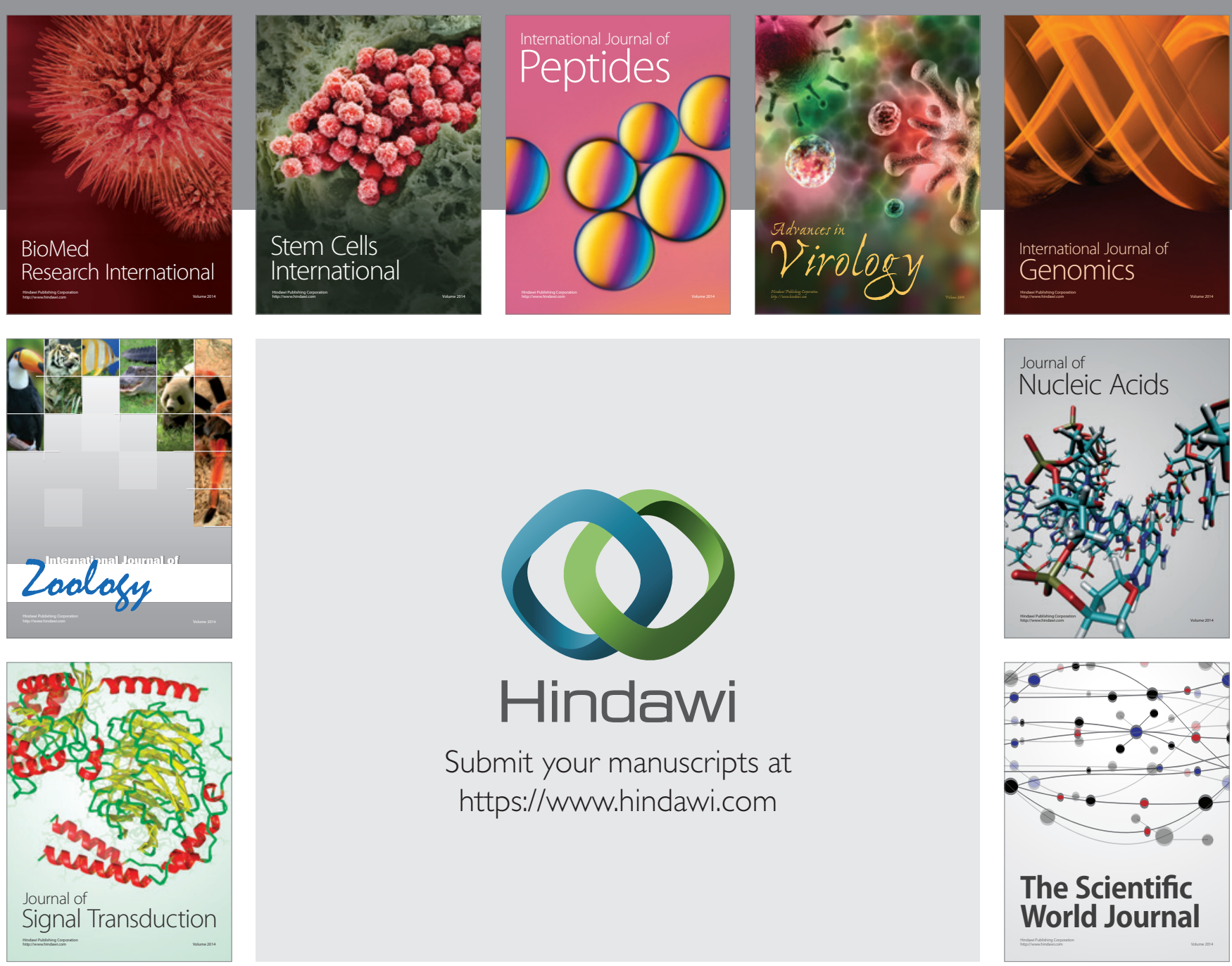

Submit your manuscripts at

https://www.hindawi.com
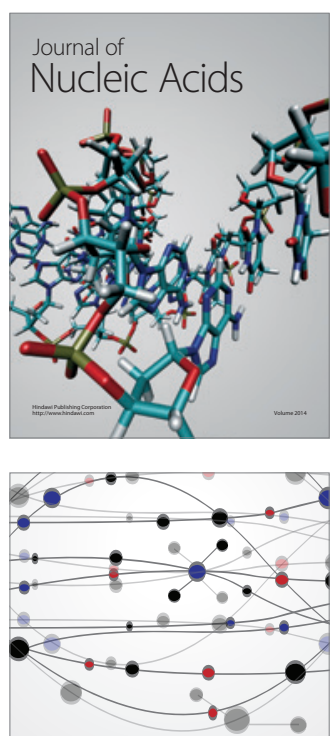

The Scientific World Journal
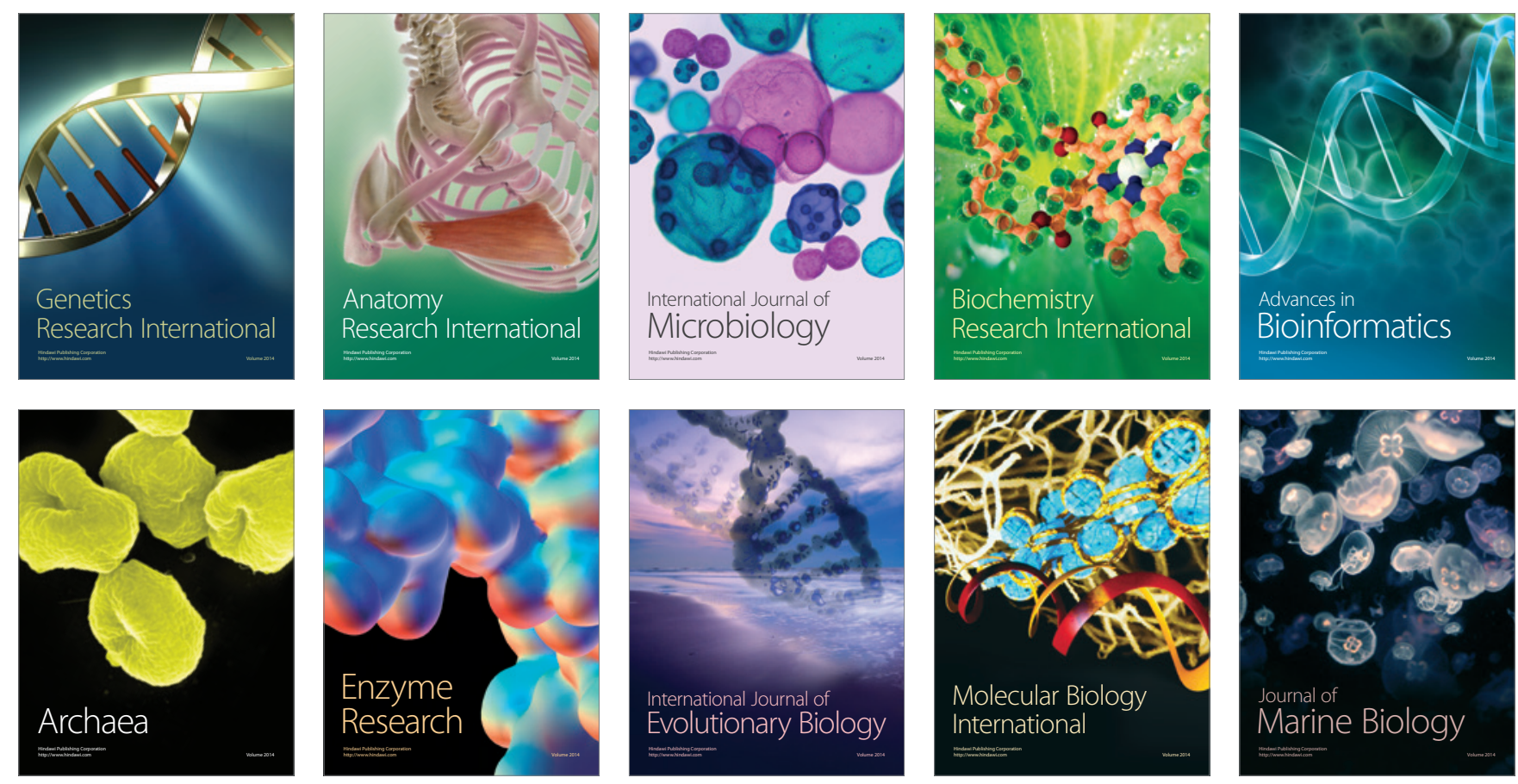